\title{
¿Cuándo una investigación se considera realmente innovadora?: Retos epistémicos en la producción de nuevos conocimientos en el derecho*
}

When is an investigation considered really innovative? Epistemic challenges in the production of new knowledge in law

Yamid Enrique Cotrina Gulfo ${ }^{1 \times}$

Fecha correspondencia:

Recibido: 28 de marzo de 2020. Revisión: 28 de abril de 2020. Aceptado: 18 de mayo de 2020

\section{Forma de citar:}

Cotrina, Yamid Enrique. "¿Cuándo una investigación se considera realmente innovadora?: Retos epistémicos en la producción de nuevos conocimientos en el derecho." En: Revista CES Derecho. Vol. 11, No. 2, junio a diciembre de 2020, 41-49.

Open access

Términos de uso

Licencia creative commons

Etica de publicaciones

Revisión por pares

Gestión por Open Journal System DOl: http://dx.doi.org/10.21615/ cesder.11.2.3

ISSN: 2145-7719

Sobre el autor:

1. Docente (Universidad del Atlántico) Abogado, Máster en Estudios Avanzados en Derechos Humanos (Universidad Carlos III de Madrid), Máster en Derecho Constitucional (Centro de Estudios Políticos y Constitucionales).

\section{Resumen}

La innovación es un criterio de calidad investigativa que ha tenido una pretensión generalizada en toda la comunidad académica; es exigido por las redes de investigación y corresponde a un factor de competitividad que se tiene en cuenta en la selección y priorización de proyectos. El proceso de investigación se constituye de responder sucesivas preguntas que corresponden a diversos elementos, entre los más importantes: el objeto de estudio, el objetivo general y la metodología. Estos elementos guían la investigación y responden respectivamente, los interrogantes de: qué se investiga, hasta qué punto y cómo se investiga un fenómeno particular. El componente innovador siempre se ha exigido desde la delimitación temática, no obstante, la metodología como etapa del proceso de investigación es la que evidencia la orientación de los productos generados y puede considerarse un indicador más relevante al momento de determinar la pertinencia y la innovación del proyecto investigativo.

Palabras clave: Epistemología, Ciencias Sociales, Conocimiento, Innovación, Investigación.

\section{Abstract}

Innovation is a criterion of research quality that has been widely sought by the entire academic community; it is required by research networks and corresponds to a competitiveness factor that is taken into account in the selection and prioritization of projects. The research process is made up of answering successive questions that correspond to various elements, among the most important: the object of study, the general objective and the methodology. These elements guide the research and answer, respectively, the questions of: what is being researched, to what extent and how a particular phenomenon is researched. The innovative component has always been demanded from the thematic delimitation, however, the methodology, as the stage of the research process is the one that evidences the orientation of the products generated, can be considered a more relevant indicator when determining the relevance and innovation of the research project. 
Keywords: Epistemology, Social Sciences, Knowledge, Innovation, Research.

\section{Introducción}

Con la frase: "Revolucionario en las ideas, liberal en los objetivos y conservador en los métodos" el político Puertorriqueño Luis Ferré (1904-2003), hizo una reflexión sobre la gestión pública en cuanto a las políticas de gobierno que llevó a cabo a lo largo de su gobierno en la isla. Toda iniciativa (idea), que se convertiría en una acción de gobierno debía partir de la premisa de generar un cambio que repercutiera en el mejoramiento de las condiciones de vida de las comunidades, de ahí derivaba el epíteto necesario de revolucionaria para toda decisión que conllevara una puesta en marcha en la realidad política y social.

Los objetivos, considerados como las metas fijadas en las políticas gubernamentales formuladas, son tenidos en cuenta como indicadores de evaluación, monitoreo y cumplimiento de las iniciativas estatales previamente reguladas, en aras de abarcar el mayor ámbito posible de impacto en términos de resultados, según Ferré, se debe tener una consciencia liberal para poder tener un mayor espectro en la definición del alcance en procura de la optimización de los recursos en términos de efectividad (eficiencia: uso de menores recursos para mayores resultados; eficacia: cumplimiento de objetivos fijados).

En términos políticos, los métodos son el aspecto más temido en términos de función pública, con el fin de no alterar el clima democrático al interior de un país, no ejercer con arbitrariedad las funciones propias de un sector ajeno al de sus competencias, y la más importante. Evitar que el postulado de Maquiavelo se imponga en la gestión de lo público: "sucede que cuando le acusan los hechos, le excusan los resultados", la cual se ha reproducido masivamente como "el fin justifica los medios".

Es imperante trasladar este pensamiento político al ámbito de la investigación teniendo en cuenta los tres aspectos a lo que Ferré se refiere en su frase célebre:

\section{Tema de investigación como idea}

El germen del objeto de estudio que se encarna como título de investigación, es el que contiene el aporte al conocimiento científico y lo que tradicionalmente en la comunidad académica pone su detenida atención frente a lo que pretende exponer el investigador.

\section{Objetivo de investigación como metas}

Lo que se quiere alcanzar con la ejecución del proyecto en términos de entrega de productos y generación de impacto en resultados. Desde el punto de vista epistémico, los objetivos de la investigación hacen referencia al cuanto se quiere agotar el objeto de estudio en relación al área del conocimiento que corresponde a su delimitación de contenido. Por tal razón, la pregunta a responder en esta fase del proceso de investigación científica es ¿Cuánto se quiere investigar? Sí se quiere ser más preciso, podría reformularse la pregunta en términos de delimitación temática a ¿Hasta qué punto se quiere investigar?

\section{Metodología de investigación como proceso}

Según Carlos Sabino, la investigación obedece a ser un proceso, toda vez que en esta se responden múltiples preguntas de manera simultánea en el transcurso de la ejecución de su fin principal, definido en la generación de nuevo conocimiento 
(Sabino, 2014). Es por ello que, de forma sistemática, se entiende a la investigación como un todo que se descompone en cada una de sus partes, que corresponden a ser indivisible y complementaria, es decir: no se puede llevar a cabo la investigación sin que se defina cada uno de sus segmentos, así como tampoco pueden operar estas partes por separado.

Retomando la frase de Ferré incluida en el epígrafe del presente trabajo, se propone una alteración de factores para valorar positivamente en términos de innovación los productos de investigación, en una dinámica que deje de darse prelación al tema de investigación como único indicador de generación de nuevo conocimiento, sino también a los nuevos métodos empleados en el proceso de investigación. En este caso, la premisa propuesta a continuación es la siguiente: Liberal en las ideas, conservador en los objetivos y revolucionario en los métodos.

\section{El salto de las ideas revolucionarias a la libertad en las ideas}

El rigor evaluativo de los productos de investigación tradicionalmente se centra en el objeto de investigación, en la temática a desarrollar. La innovación entonces se tiene en cuenta como el indicador de excelencia académica por antonomasia, sin embargo, luego del avance investigativo, la multiplicidad de actores intervinientes en la comunidad científica y la multiplicidad de actores que ponen a consideración sus iniciativas de investigación hace que innovar desde la formulación de un tema de investigación que resulte novedoso a la luz de su originalidad, sea un desafío.

La democratización del conocimiento evidenciada en la masificación de los productos de Ciencia y Tecnología desde las instituciones de educación superior, ha generado nuevos procesos de generación de conocimiento en las dinámicas de Ciencia, Tecnología y Sociedad reflejados en productos de investigación derivados de iniciativas académicas (proyectos de investigación), con la particularidad de que los indicadores de pertinencia del proyecto son su utilidad práctica y su proyección social. (Alonso, 2008).

Las ideas liberales en este caso se refieren a la incursión a la interdisciplinariedad e incluso, a la pretendida transdisciplinariedad como paradigma epistémico emergente. Autores como Barasab Nicolescu afirma "La transdisciplinariedad concierne (...) lo que está a la vez entre las disciplinas, a través de las diferentes disciplinas y más allá de toda disciplina. Su finalidad es la comprehensión del mundo presente en el cual uno de los imperativos es la unidad del conocimiento". (Nicolescu, 1996, p. 35)

La innovación como necesidad urgente en las ciencias sociales, se debe al estancamiento epistémico que tienen estas disciplinas a causa del abuso del paradigma histórico-hermenéutico frente al uso del paradigma empírico-analítico de manera generalizada en las ciencias exactas. (Bunge, 1997). El positivismo trajo consigo el actual paradigma de "como conocer" el derecho, entre ellos se destacan los "necesarios" reduccionismos que se planteaban desde la lógica jurídica para dar validez al conocimiento generado en ella. Toda vez que consistía en la separación del objeto de conocimiento y el sujeto cognoscente para dar consigo la "objetividad" pretendida. Esto significó la necesidad de un cambio metodológico en el paradigma de esta disciplina, lo cual derivó en una fuerte crisis del positivismo, abriendo la puerta a enfoques epistémicos alternativos que dieran un salto del dualismo empírico-analítico de las ciencias exactas e histórico-hermenéutico de las ciencias sociales, hacia el constructivismo jurídico como paradigma de investigación a través del enfoque crítico-social. 
Diluir los campos de investigación, que definen las disciplinas como unidades epistémicas compartimentadas, para darles paso a los campos de estudio e ideas libres que dejen de estar condicionadas a las barreras propias de las disciplinas, es el punto inicial para llegar a la interdisciplinaridad y este a su vez conlleva a la transdisciplinariedad utilizando variables compuestas o métodos propios de una u otra disciplina. (Pérez y Setien, 2008).

\section{De los objetivos tiberales a los objetivos conservadores}

Desde el punto de vista epistémico, los objetivos de la investigación hacen referencia al cuanto se quiere agotar el objeto de estudio en relación al área del conocimiento que corresponde a su delimitación de contenido. Por tal razón, la pregunta a responder en esta fase del proceso de investigación científica es ¿Cuánto se quiere investigar? Sí se quiere ser más preciso, podría reformularse la pregunta en términos de delimitación temática a ¿Hasta qué punto se quiere investigar? La investigación en este ámbito expone dos dimensiones que confluyen.

La dimensión subjetiva de la investigación, se centra en la fase preliminar y de formulación del proyecto de investigación: escoger el tema de investigación, delimitarlo, seleccionar el objeto de investigación y definir los métodos para su debido agotamiento. En este momento el investigador, investido por su discrecionalidad de definir el "¿Qué?" y “¿Cómo?" lo quiere investigar, sienta las bases para la ejecución de un proyecto de investigación.

La dimensión objetiva de la investigación, por el contrario, se desprende de la discrecionalidad del investigador y por lo tanto los productos derivados del proceso de investigación pasan a depender de la interacción con el contexto aplicable, fundamentado en el componente teórico vinculado al objeto de estudio. Cualquier intervención del investigador frente a la obtención de resultados será considerada como tendenciosa y supondrá un sesgo que será un obstáculo para la generación de conocimiento científico, especialmente en su universalidad.

Desde una perspectiva funcionalista, el objetivo general puede ser visto de las siguientes maneras:

\section{Horizonte de finalización}

Hace de las veces del límite al que el investigador en términos de delimitación temática o de contenido, el objetivo general expresa hasta cuándo se debe investigar. Es decir, representa una frontera de conocimiento.

\section{Premisa Mayor}

Todos los productos de investigación derivados de la ejecución de las actividades definidas, deben estar sujetos a la guía contemplada en la afirmación precisa que representa el objetivo general, la cual rige el sentido de todo lo que se ejecuta en el contexto del proyecto, actúa como el encuadre metodológico en el que se delimita el conocimiento a generar.

\section{Faro hermenéutico}

Siguiendo el caso de la categoría anterior, además de que el objetivo general supone el condicionamiento de un contexto epistémico, también hace lo suyo en el desarrollo argumentativo e interpretativo de cada uno de los avances que se tenga en la investigación. 
Los objetivos deben ser medibles, verificables y alcanzables, en este último criterio se destaca la característica conservadora de esta fase del proceso de investigación. Debe centrarse en el horizonte de finalización del objeto de estudio en el sentido epistémico (hasta donde abarcar) y temporal (hasta cuando abarcar). Los objetivos son entonces, los límites de la investigación y estos deben ser trazados de una manera concreta y ajustadas a las posibilidades reales del investigador para agotar el objeto de estudio. (Hurtado, 2005).

\section{El giro epistémico de los métodos conservadores a los métodos revolucionarios}

La innovación en las investigaciones no solo debe de partir en la originalidad del tema seleccionado a tratar, también, de la manera en cómo se aborda la temática respondiendo al ¿Cómo?, de la investigación. Cuando se menciona la metodología, no solo se refiere al diseño metodológico, como es el caso de la paradigma, tipo y fuentes de investigación; sino también al procedimiento de investigación. El cual consiste en la aplicación del método de investigación, como es el caso del análisis, la síntesis, el método histórico e incluso la hermenéutica.

Generalmente, las redes de investigación a través de sus evaluadores exigen a los investigadores métodos conservadores para la ejecución del proyecto, en el sentido de exigir una coherencia en cada uno de los elementos del diseño metodológico. El error, consiste en que solo se tenga en cuenta una coherencia estándar y no una evaluación aplicable a cada caso concreto. (Krause, 1995). Cuando se permite ampliar el margen de maniobra del investigador para incluir métodos que le son académicamente propios a otras disciplinas, se obtienen resultados novedosos, aun cuando se traten de temáticas ya abordadas y con amplio desarrollo bibliográfico.

En las ciencias sociales, el caso más preocupante de estancamiento epistemológico lo puede estar atravesando el Derecho, puesto que existe un diseño metodológico que varía muy poco y los procesos de investigación resultan ser muy poco dinámicos. (Fariñas, 1994).

Su paradigma de investigación predominante es el histórico-hermenéutico, siendo el crítico-social, de aplicación incipiente. (Melero, 2012). El uso alternativo de las herramientas metodológicas en función a la consecución de productos de investigación que signifiquen no solo un aporte real al conocimiento científico, sino que también sea innovador en la comunidad académica y cuente con innovación social.

Es por ello que se debe profundizar en la justificación práctica del proyecto y responder a la pregunta ¿A quién le sirven los productos de la investigación? Los beneficiarios de los proyectos de investigación en general, son los miembros de la sociedad, y la investigación debe ser entonces considerada como el medio para generar un proceso de apropiación social del conocimiento. Esto se obtiene mediante el diseño de procedimientos de investigación que generen mayor impacto en esa área que se desea conocer, por consiguiente, obteniendo así el fin pretendido con el uso alternativo de la investigación, más que generar un cúmulo de artículos o ponencias, también es incidir positivamente, poniendo el conocimiento al servicio público.

El paso de los métodos conservadores a los métodos revolucionarios empieza por cambiar las acciones académicas, para obtener nuevos productos investigación, que a su vez generen nuevos y mejores resultados en términos de impacto. 
Se habla de giro hermenéutico, al pretender superar los paradigmas que tratan de compartimentar el conocimiento, no solo en disciplinas, sino en maneras de obtenerlo. Rotulando a una disciplina con un paradigma propio de investigación, un diseño metodológico propio de investigación y un procedimiento propio de investigación.

La pretensión transdisciplinar recobra de nuevo sentido al emplear métodos de uno y otro campo de investigación para obtener productos de investigación beneficioso para la ciencia y la sociedad en su conjunto.

\section{Investigación jurídica integrativa como medio para la comprensión holística de los problemas jurídicos}

El uso de metodologías mixtas y de paradigmas que parecen ser propios de otras disciplinas es reconocido por voces de la comunidad jurídica, de los cuales se pueden destacar críticas al actual estado de crisis por el cual atraviesa la investigación en derecho. Entre ellas se destacan "El predominio de la concepción positivista-formalista del derecho; El aislamiento de los estudios jurídicos respecto al resto de las ciencias sociales que impiden enfoques globales a los problemas jurídicos". (Witker, 2008, p. 943) Esto ha traído notables consecuencias en la enseñanza del derecho, entre las cuales se destaca "una enseñanza jurídica discursiva, memorística y repetitiva que omite todo juicio crítico y participativo de estudiantes pasivos y especialmente receptivos". (García, 1990, p. 88)".

Tal como lo enuncia Witker, se hace necesaria una apertura al nuevo conocimiento y a otras formas de conocer superando los paradigmas de estudios heredados de los años 30 del siglo pasado. Generando así una discusión respecto al conocimiento jurídico desde los retos que suponen las necesidades de la sociedad actual. (Rodríguez, 2006). Este aislamiento fue producto de la fijación por "predeterminados" a cierto diseño metodológico que se aplica en el derecho por un amplio consenso de la comunidad jurídica, producto del positivismo-formalista: el paradigma histórico-hermenéutico.

El derecho ha pasado a comprender los fenómenos desde la hermenéutica sin ir más allá de los fallos que son generados con su adecuación a las dinámicas sociales. Es por ello que la evolución del derecho, por lo general está detrás de los cambios experimentados en la sociedad y no es ese medio por el cual se puede efectuar un cambio en el contexto aplicable. Aquí puede confirmarse entonces, que el derecho se adecúa a la sociedad, y este a dejado de ser ese instrumento que condiciona la sociedad. Tal como lo expresa Atienza "El Derecho, en cuanto fenómeno social e histórico, es evidentemente, una obra humana, pero en cierto sentido, adquiere vida propia." (Atienza, 1998, p. 23).

Aunque no pueda prever los cambios sociales, anticiparse a ellos y regularles antes que lo demanden, todo esto, producto del formalismo en el que la hermenéutica ha tenido un rol transversal en cuanto a la aplicación del derecho. Solamente se enfoca en los sucesos en pleno desarrollo, sin poder adelantarse a aquellos fenómenos que emergen producto de los cambios sociales.

La vinculación del derecho con otras disciplinas puede ser visto desde el estudio interdisciplinario, pero también desde la incorporación de paradigmas, métodos y técnicas de investigación, que se suponen "propios" de otras ciencias. Con el fin de 
evitar caer en esa constante dinámica de "sistematizar normas jurídicas positivas para facilitar la interpretación y aplicación del Derecho”. (Witker, 1994, p. 82).

La aplicación del "método propio" del derecho, que ha generado ese enunciado estancamiento en cuanto a la generación de nuevos conocimientos, tiene su explicación en la teoría kelseniana, citando a Bobbio en el que "la dogmática jurídica, dentro de este paradigma, es triplemente normativa: a) Objeto, b) Método y c) Función. Así el objeto lo constituyen las fuentes formales y sus significados; el método, la hermenéutica en su interpretación y aplicación, y la función referida a los efectos que la interpretación-aplicación de las normas tiene sobre la realidad social". (Kelsen, 1983, p. 83)

En este sentido, se fijó un paradigma como válido que ha sido reproducido sistemáticamente en la investigación jurídica, tal es el caso de la visión positivista formalista, la cual ha pretendido generar aquella ilusión de objetividad en la que el observador se encuentre completamente separado del objeto a investigar. Es por ello que la visión crítica del investigador se encuentra ausente, lo cual le permitiría anticiparse a los hechos sociales emergentes, que requieren ser regulados para una mejor garantía en el contexto del Estado de Derecho, sin embargo, este criterio era interpretado como un sesgo y se percibía como un elemento que restaba rigurosidad al producto derivado de la investigación.

Es por ello que superar el formalismo-positivista como paradigma predominante, mediante la inclusión de otras formas de entender el derecho aplicadas desde otras disciplinas puede contribuir a una comprensión holística de la investigación jurídica y sociojurídica. Superando la hermenéutica como única forma conocer el derecho, incorporando métodos que son aplicados en otras ramas del saber, hace entonces que se cuente con un campo de acción de mayor impacto en el proceso de investigación jurídica y esto se refleje en un uso alternativo del derecho, tal como lo desarrollan teóricamente las critical legal studies, incorporando una visión transformadora del derecho en cuanto a su pertinencia como disciplina en la sociedad y sus dinámicas de cambio.

\section{Conclusiones}

La innovación exigida en las ideas para plantear temas de investigación novedosos, ha impactado significativamente la producción académica de las ciencias sociales, esto ha implicado que se produzca un estancamiento epistemológico, en la medida que no se permita variar los métodos para obtener productos de investigación que varíen a la tendencia actual que maneja la comunidad académica.

La incidencia del positivismo como paradigma hegemónico en las ciencias, ha hecho que se otorgue prelación a aquellas disciplinas que generan productos de investigación desde la concepción empírico-analítica del conocimiento. Esto se traduce en la preferencia de las políticas de financiamiento a la Ciencia, Tecnología e Innovación a las disciplinas propias de las ciencias exactas (Lucio, 2013).

Los objetivos conservadores hacen que sean alcanzables, esta cualidad en el proceso de investigación, se refleja en cuanto las metas trazadas por el investigador son medibles y alcanzables. Definir objetivos, es la acción en la que se debe tener mayor cautela en cuanto al horizonte de finalización y la delimitación temática. Las acciones direccionadas al cumplimiento de los objetivos de investigación, son las actividades definidas en el cronograma y deben constituir una secuencia de pasos 
para la entrega de productos y generación de resultados de investigación en términos de impacto.

Los métodos conservadores son los que han generado el actual estancamiento en las ciencias sociales en cuanto a la generación de nuevos conocimientos. Puesto que, en un solo sentido que se considera estándar y únicamente valido por ser el mayormente aceptado en la comunidad académica, de igual manera se ha impuesto una única coherencia metodológica en cuanto al diseño de la investigación: paradigma, tipo, método y fuentes; el cual hace que estas ciencias sean cada vez más estáticas.

Caso contrario, sucede con los métodos revolucionarios, propios de disolver las fronteras epistémicas y acabar con los paradigmas vedados para otras disciplinas. También, implica en el planteamiento de nuevos diseños metodológicos que incluyan paradigma, tipo, método y fuentes de investigación que si bien, pueda que se alejen de ese consenso de lo correctamente investigable, constituye una verdadera innovación en cuando a la generación de productos de investigación (artículos, libros, ponencias, etc.).

La premisa propuesta: liberal en las ideas, conservador en los objetivos y revolucionario en los métodos, en contraposición con la expuesta en la frase de epígrafe. Dado a lo expuesto con anterioridad puede significar la consigna que sustente y aliente una nueva generación de investigadores con visiones y perspectivas del conocimiento, más abiertas que los reduccionismos positivistas para darle paso al uso alternativo de la investigación propuesto anteriormente, que consiste en ponderar la innovación social como criterio de innovación científica en el derecho.

\section{Referencias}

Barrio Alonso, Cipriano. (2008) La apropiación social de la ciencia: nuevas formas. CTS: Revista iberoamericana de ciencia, tecnología y sociedad, vol. 4, no 10, p. 213-225.

Bunge, Mario. (1997) Epistemología: curso de actualización. Siglo XXI, Buenos Aires.

Fariñas Dulce, María José. (1994) Sociología del Derecho Versus Análisis sociológico del derecho, Cuadernos de Filosofía del Derecho. Núm. 15-16, p, 1013-1023.

García Garrido, Manuel y Eugenio Francisco (1990), Estudios de derecho y formación del jurista, Madrid, Dikinson.

Hurtado de la Barrera, Jacqueline, (2005) Como formular objetivos de investigación, Ediciones Quirón, Caracas (Venezuela).

Kelsen, Hans, (1983) Teoría pura del derecho, trad. de Roberto Venengo, México, UNAM.

Krause, Mariane. (1995) La investigación cualitativa: un campo de posibilidades y desafíos. Revista Temas de educación, vol. 7, no 7, p. 19-40.

Lucio, Jorge. (2013) Ciencia y tecnología en la universidad colombiana. COLCIENCIAS, Bogotá.

Melero Aguilar, Noelia. (2012) El paradigma crítico y los aportes de la investigación acción participativa en la transformación de la realidad: un análisis desde las ciencias sociales. Cuestiones pedagógicas, 21, 339-355.

Nicolescu, Barasab, (1996) La transdisciplinariedad: Un manifiesto. Mónaco, Rocher.

Pérez Matos, Nuria Esther y Setien Quesada, Emilio. (2008) La interdisciplinariedad y la transdisciplinariedad en las ciencias: una mirada a la teoría bibliológico-informativa. ACIMED [online], vol.18, n.4 Disponible en: <http://scielo.sld.cu/scielo. php? script=sci arttext\&pid $=$ S1024-94352008001000003 $>$. Citado el 18 de junio de 2019. 
Pág 49

Rodríguez Cepeda, Bartolo Paolo. (2006) Metodología Jurídica. Oxford University Press, Ciudad de México.

Sabino, Carlos. (2014) El proceso de investigación. Editorial Episteme, Guatemala. Witker, Jorge, (2008) Hacia una investigación jurídica Integrativa. Boletín Mexicano de Derecho Comparado, nueva serie, año XLI, núm. 122, mayo-agosto, pp. 943-964. , (1994) La investigación jurídica, México, McGraw-Hill. 